\title{
Neuer Marker zur Einschätzung der „sicheren Entlassung“
}

\author{
Jeder 5. Patient, der eine akute Exazerbation der COPD (AECOPD) \\ überlebt und aus der Klinik entlassen wird, muss innerhalb von \\ 4 Wochen erneut wegen einer akuten Exazerbation hospitalisiert \\ werden. Die Messung des neuralen Atemantriebs, des Verhältnisses \\ von Atemmuskellast und -kapazität soll helfen, rezidivgefährdete \\ Patienten schon vor der Entlassung zu identifizieren. \\ Thorax 2015; 70: 1123-1130
}

Die beidseitige Elektromyografie des Musculus intercostalis parasternalis in Höhe des 2. Interkostalraums ( $\left.\mathrm{EMG}_{\text {para }}\right)$ ist nicht nur transösophageal, sondern neuerdings auch transdermal und damit nicht-invasiv möglich. Dies haben sich die Londoner Forscher um E.-S. Suh für eine monozentrische prospektive Beobachtungsstudie zunutze gemacht: Sie schlossen 120 hospitalisierte AECOPD-Patienten in ihre Studie ein und erfassten neben den üblichen $\mathrm{Vi}$ tal- und Spirometrieparametern (FVC, $\mathrm{FEV}_{1}$, IC) und der Symptomatik auch die elektrische Spannung des oberflächlichen Muskels. Zudem wurde der Krankheitsverlauf beurteilt.

$\mathrm{EMG}_{\text {para }}$ wurde mind. 1-mal täglich gemessen; der Wert wurde ins Verhältnis zu einem einmalig gemessenen $\mathrm{EMG}_{\text {para }}$ während eines maximal forcierten Inspirationsmanövers $\left(\mathrm{EMG}_{\text {para\%max }}\right)$ gesetzt. Das Produkt aus EMG paramax $_{\text {max }}$ und der Respirationsrate ergibt den neuralen Atemflussindex (Neural Respiratory Drive Index, NRDI). Eine eigens entwickelte Software wertete die Befunde automatisch aus und stellte dabei die Werte bei Einlieferung und kurz vor Entlassung gegenüber. So wurden $\mathrm{EMG}_{\text {parammax }}$ und der NRDI fortlaufend mit ihren Nachfolgewerten verglichen $\left(\triangle \mathrm{EMG}_{\mathrm{para} \text { max,cons }}, \Delta \mathrm{NRDI}_{\text {cons }}\right)$.

Als Cut-off für eine aussagekräftige Verbesserung von $\mathrm{EMG}_{\text {para\%max }}$ wurde eine Absenkung des Werts um mindestens 3,1\% über die Zeit des Klinikaufenthalts festgelegt ( $\triangle E M G_{\text {paramax }} \leq 3,1 \%$ ). $\mathrm{D}$, dies ergab sich aus früheren Studien. Die Symptomverbesserung der Patienten schlug sich in steigenden Spirometriewerten, sinkenden Werten der Symptomskalen (modifizierte Borg-Skala, COPD-Assessment-Test (CAT) sowie in verringerten Werten für $\mathrm{EMG}_{\text {para\%max }}$ und NRDI nieder. $\triangle \mathrm{EMG}_{\text {para\%max }}$ war deutlich invers korreliert mit $\triangle \mathrm{FEV}_{1}, \Delta \mathrm{FVC}$ und $\triangle \mathrm{IC}$, allerdings nicht korreliert mit den Veränderungen der Borg- und CAT-Werte über die Zeit des Klinikaufenthalts.

Es gab 116 Fälle von klinischer Verschlechterung (mind. 1 Punkt Anstieg in der BorgSkala) und 35 Fälle, in denen die Mediziner eine Verschlechterung feststellten. Dies war i. d. R. assoziiert mit einem An-

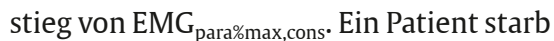
3 Tage nach Klinikentlassung; die Rehospitalisierungsrate lag nach 14 Tagen bei $12,5 \%$ und nach 28 Tagen bei $21,7 \%$.

Sowohl $\triangle E M G_{\text {paramax }}$ als auch $\triangle$ NRDI waren prädiktiv für eine Wiedereinweisung innerhalb von 14 Tagen. So war bei Patienten, deren $\mathrm{EMG}_{\text {para\%max }}$ um mind. 3,1\% gefallen war, das Risiko der Wiedereinweisung gering; der negative prädiktive Wert betrug $97,7 \%$. Der positive prädiktive Wert für eine erneute Hospitalisierung beim Ausbleiben einer so deutlichen Verbesserung lag allerdings nur bei $19,7 \%$. Dementsprechend war die Sensitivität des Tests hoch (93,8\%), die Spezifität nicht (41,3\%).

\section{Fazit}

Bei der Wiedereinweisung innerhalb von 28 Tagen spielten nach Angaben der Autoren noch andere Faktoren eine wichtige Rolle, etwa das Alter. Zumindest für Patienten unter 85 Jahren waren die neuen Marker aber auch über 28 Tage aussagekräftig. Der Cut-off-Wert von 3,1\% für $\triangle \mathrm{EMG}_{\mathrm{para} \text { max }}$ soll in weiteren Studien validiert werden.

Simone Reisdorf, Erfurt 